\title{
Globe
}

Revue internationale d'études québécoises

\section{La politique du multiculturalisme est-elle compatible avec le nationalisme québécois? Is Multicultural Politics Compatible with Quebecois Nationalism?}

\section{Stéphane Courtois}

Volume 10, numéro 1, 2007

Étranger et territorialité

URI : https://id.erudit.org/iderudit/1000079ar

DOI : https://doi.org/10.7202/1000079ar

Aller au sommaire du numéro

Éditeur(s)

Globe, Revue internationale d'études québécoises

ISSN

1481-5869 (imprimé)

1923-8231 (numérique)

Découvrir la revue

Citer cet article

Courtois, S. (2007). La politique du multiculturalisme est-elle compatible avec le nationalisme québécois? Globe, 10(1), 53-72. https://doi.org/10.7202/1000079ar
Résumé de l'article

Dans ce texte, l'auteur entend montrer que le nationalisme québécois ne s'oppose en rien à une politique de multiculturalisme. Dans la première partie, il fait valoir que ce que le nationalisme québécois rejette n’est pas le multiculturalisme en tant que tel, mais le type de multiculturalisme promu à l'intérieur de la fédération canadienne, qui peine à reconnaître la composition multinationale du Canada. Dans la seconde partie, il tente de répondre à l'objection selon laquelle nationalisme québécois et multiculturalisme seraient des options conflictuelles, non en raison de la politique canadienne sur le multiculturalisme, mais à cause du nationalisme québécois lui-même, qui, en voulant préserver l'identité nationale des Québécois, limiterait le degré d'ouverture du Québec à la diversité culturelle. Ce texte cherche à prouver que cette position n'est pas fondée. 


\title{
LA POLITIQUE \\ DU MULTICULTURALISME \\ EST-ELLE COMPATIBLE \\ AVEC LE NATIONALISME \\ QUÉBÉCOIS?
}

\author{
STÉPHANE COURTOIS \\ Université du Québec à Trois-Rivières
}

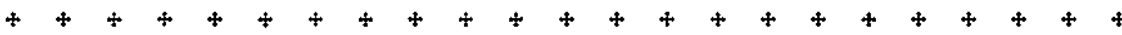
Résumé - Dans ce texte, l'auteur entend montrer que le nationalisme québécois ne s'oppose en rien à une politique de multiculturalisme. Dans la première partie, il fait valoir que ce que le nationalisme québécois rejette n'est pas le multiculturalisme en tant que tel, mais le type de multiculturalisme promu à l'intérieur de la fédération canadienne, qui peine à reconnaître la composition multinationale du Canada. Dans la seconde partie, il tente de répondre à l'objection selon laquelle nationalisme québécois et multiculturalisme seraient des options conflictuelles, non en raison de la politique canadienne sur le multiculturalisme, mais à cause du nationalisme québécois lui-même, qui, en voulant préserver l'identité nationale des Québécois, limiterait le degré d'ouverture du Québec à la diversité culturelle. Ce texte cherche à prouver que cette position n'est pas fondée.

\section{Is Multicultural Politics Compatible with Quebecois Nationalism?} Abstract - In this text, the author intends to show that Quebecois nationalism is in no way opposed to a politics of multiculturalism. In the first part, he emphasizes that what Quebecois nationalism rejects is not multiculturalism as such, but rather the type of multiculturalism promoted within the Canadian federation, insofar as it resists recognizing the multinational composition of Canada. In the second part, he attempts to respond to the objection according to which Quebecois nationalism and multiculturalism would be conflicting options not by virtue of Canadian policy on multicuturalism, but because of Quebecois nationalism itself, which, in guarding the national identity of Quebecois people, would limit Quebec's degree of openness to cultural diversity. This text intends to show that that position has no grounds.

$++4+\div+4+4+4+4+4+4+4+4+4$ GLOBE. REVUE INTERNATIONALE D'ÉTUDES QUébécoises - VOLUME IO - NUMÉro I - 2007 
Lors de son passage à une série d'émissions télévisées ${ }^{1}$ où des personnalités publiques étaient invitées à réagir au documentaire Point de rupture (sur le référendum de 1995), l'ex-ministre libéral des Affaires étrangères du Canada Pierre Pettigrew a fait valoir ce qui, de son point de vue, constitue le véritable défi du nationalisme québécois: il s'agirait d'un projet en concurrence avec le multiculturalisme canadien, puisqu'il mettrait en cause le modèle d'intégration de la diversité valorisé au Canada, axé sur la tolérance, le pluralisme et l'ouverture aux différences culturelles. L'alternative suggérée par l'ex-ministre libéral semblait être la suivante: ou bien le nationalisme québécois devient de plus en plus tolérant et ouvert à la diversité, comme tendraient à le prouver la condamnation presque générale des propos tenus par Jacques Parizeau lors du dernier référendum ainsi que les positions officielles adoptées depuis ce jour par tous les porte-parole du mouvement souverainiste québécois (Lucien Bouchard, Bernard Landry, Gilles Duceppe, etc.) - mais alors la politique québécoise sur le multiculturalisme se distingue de moins en moins de la politique canadienne et le nationalisme québécois devient sans objet - ou bien le nationalisme québécois se définit en opposition au multiculturalisme canadien. Celui-ci a en effet été longtemps conspué par les souverainistes en raison de son inaptitude à reconnaître la spécificité québécoise. Toutefois, le nationalisme québécois devient alors peu attrayant puisqu'il semble contester les valeurs, chères à une multitude de Canadiens et de Québécois, de tolérance, d'ouverture et de respect des différences. En un mot, multiculturalisme et nationalisme (entendons: un certain type de nationalisme, le nationalisme québécois) seraient incompatibles. J'aimerais dans ce texte utiliser cette sortie de l'ex-ministre libéral, qui est pratiquement passée inaperçue dans les médias ${ }^{2}$, pour rejeter ce type de position et montrer son inadéquation à la réalité politique actuelle au Québec. Si cette position a sans doute été soutenue à des fins polémiques dans le contexte d'un débat autour du documentaire Point de rupture, elle n'en traduit pas moins une certaine perception du nationalisme québécois fermement ancrée chez plusieurs observateurs et analystes, tant au Québec qu'au Canada, qui

$$
++
$$

1. J'a à l'esprit l'émission La part des choses du 9seprembre 2005, où Pierre Pettigrew étair confronté à l'ex-président du Conseil du rrésor et ministre d'État à l'Administration ex à la Fonction publique Joseph Facal, ainsi que l'émission $L e$ Potnt, égalemenr du 9septembre 2005, où il avait comme vis-à-vis l'ex-ministre des Relatıons internationales du Québec Louise Beaudoin.

2. Ceux-ci se sont en effet concentrés sur le rejet par le ministre Petcigrew de la doctrine Gérin-Lajoie. En réaction à certe position, voir par exemple l'article signé conjointement par le minustre québecois des Affaires intergouvernementales Benoît Pelletier et la ministre québécoise des Relations internationales MoniqueGagnon-Tremblay dans Le Devoir, 10et 11 septembre 2005, http://www.ledevorr.com/2005/09/10/90040.html (28 août 2007). 
m'apparât mal fondée pour un ensemble de raisons qui seront présentées dans les lignes qui suivent.

\section{LE REJET DU MULTICULTURALISME CANADIEN}

Une première clarification s'impose au sujet du rapport qu'entretient le Québec depuis une vingtaine d'années avec le multiculturalisme canadien, disons depuis le rapatriement de la constitution par Pierre Elliott Trudeau en 1982. La question en jeu ici est celle du type de multiculturalisme rejeté par le Québec. En quoi exactement consiste le multiculturalisme canadien? Et qu'est-ce qui, dans le multiculturalisme canadien, fait problème au Québec?

Le multiculturalisme "canadien" est indissociable de la vision d'ensemble de la nation canadienne promue par le Parti libéral du Canada depuis Pierre Elliott Trudeau. Quatre éléments y sont étroitement imbriqués: la primauté accordée à l'identité civique canadienne, qui se greffe autour de la Constitution canadienne et de la Charte des droits et libertés qui y a été enchâssée; la politique canadienne sur le bilinguisme, selon laquelle deux langues officielles sont reconnues au Canada, le français et l'anglais; le principe de l'égalité des provinces; enfin, la politique canadienne sur le multiculturalisme, laquelle combine, d'une part, la protection et la promotion de l'identité des groupes culturels dont se compose la mosaïque canadienne et, d'autre part, les mesures facilitant l'intégration des immigrants à l'un des deux principaux groupes linguistiques reconnus au Canada.

Qu'est-ce qui fait problème dans cette vision pour la plupart des nationalistes québécois? Est-ce la Charte des droits et libertés? Le Québec dispose pourtant de sa propre charte depuis 1975, soit avant même le rapatriement de la Constitution canadienne en 1982. Ce ne sont donc pas les valeurs libérales et démocratiques de base que l'on y retrouve qui font problème puisqu'elles sont sensiblement les mêmes que celles qui figurent dans la Charte québécoise des droits et libertés de la personne. Est-ce alors la politique canadienne sur le multiculturalisme proprement dite? Les énoncés de politique sur l'immigration que l'on rencontre dans la plupart des documents officiels du gouvernement du Québec expriment, à peu de choses près, le même désir d'ouverture à la diversité ethnique et culturelle dans le respect des valeurs démocratiques fondamentales que celui qui est formulé dans la Loi sur le multiculturalisme canadien. Ainsi, par exemple, le ministère québécois de l'Immigration et des Communautés culturelles présente le Québec comme une "société d'accueil" qui reçoit les immigrants venus des quatre coins du globe avec leur savoir-faire, leurs compétences, leur langue, 
leur culture et leur religion. Le gouvernement du Québec présente également la province comme une "société pluraliste", où

la majorité d'origine française cohabite avec une minorité d'origine britannique, des nations amérindiennes et des gens de diverses origines et cultures venus d'ailleurs dans le monde. Le gouvernement du Québec considère cette diversité comme une richesse. Il encourage l'échange entre les cultures et le rapprochement entre les collectivités ${ }^{3}$.

Comme on le voit, s'il subsiste des différences entre les politiques sur le multiculturalisme adoptées au Canada et au Québec (peu importe que ces politiques s'expriment dans la Loi canadienne sur le multiculturalisme ou dans les documents officiels du gouvernement du Québec relatifs à l'immigration et aux communautés culturelles), elles semblent au premier abord plutôt ténues.

Qu'est-ce qui, alors, fait problème dans cette vision de la nation canadienne pour la plupart des nationalistes québécois? Ce n'est pas la politique canadienne sur le multiculturalisme en elle-même qui suscite la critique, mais la façon dont on doit l'interpréter à partir du moment où elle s'articule aux deux autres éléments qui composent la vision de la nation canadienne promue par Pierre Elliott Trudeau, soit la politique canadienne sur le bilinguisme et le principe de l'égalité des provinces.

La politique canadienne sur le bilinguisme, adoptée sous Trudeau en 1969, deux ans avant la politique sur le multiculturalisme, consacre une certaine vision des peuples d'origines française et britannique et de leur cohabitation au sein du Canada. Réagissant au rapport de la commission Laurendeau-Dunton sur le bilinguisme et le biculturalisme, cette politique reconnaît l'existence de deux moyens de communication officiels au Canada, le français et l'anglais. Cependant, elle élimine leur ancrage dans deux communautés d'accueil "nationales», c'est-à-dire la réalité de deux communautés politiques distinctes à l'intérieur du Canada et de deux stratégies différentes de state nation building, l'une ayant son siège dans la province de Québec et l'autre se situant dans le reste du Canada. Bref, la politique canadienne sur le bilinguisme consacre une vision strictement linguistique, et non nationale ou culturelle, des deux principales communautés d'accueil au Canada.

$$
++4
$$

3. Toutes ces informations sont disponibles sur le site officiel du ministère de l'Immigration et des Communautés culturelles du gouvernement du Québec (www.immigration-quebec.gouv.qc.ca). 
À la politique sur le bilinguisme s'ajoute le principe de l'égalité des provinces, qui représente ni plus ni moins la lecture officielle de la Constitution au sein du Canada anglais, lecture suggérée par les articles 92 et 93 de la Loi constitutionnelle de 1867 , selon lesquels toutes les provinces ont les mêmes pouvoirs juridiques et la même relation avec leurs citoyens et avec le gouvernement fédéral. $\mathrm{Si}$, donc, on combine la politique canadienne sur le bilinguisme au principe de l'égalité des provinces, il n'y a plus qu'une façon de comprendre et d'interpréter la politique canadienne sur le multiculturalisme: toutes les cultures dont se compose le Canada sont sur un même pied d'égalité, la culture nationale québécoise n'étant qu'une des multiples figures de cette mosaïque. La politique canadienne sur le multiculturalisme, à partir du moment où elle est lue à travers le prisme de la politique sur le bilinguisme et du principe de l'égalité des provinces, dissimule l'existence au Canada d'une majorité nationale anglophone et de plusieurs minorités nationales: celles formées par le Québec francophone et par les nations autochtones. En un mot, la politique canadienne sur le multiculturalisme camoufle et dissimule la réalité multinationale du Canada. Voilà essentiellement ce que les nationalistes du Québec ont à reprocher à la politique canadienne sur le multiculturalisme. Ce qui est en question, ce n'est pas l'ouverture aux différences culturelles ni la promotion de la diversité. Le Québec n'est pas moins ouvert que le Canada à la différence culturelle et à la diversité. Ce qui est en question, c'est la façon dont une telle politique peut être utilisée pour occulter une certaine réalité sociologique, à savoir l'existence d'une multitude de communautés nationales à l'intérieur du Canada et, surtout, l'existence d'un véritable État à l'intérieur de l'État canadien: l'État québécois.

De ce qui précède on peut conclure provisoirement qu'il est faux de prétendre que le nationalisme québécois ne s'accommode pas du multiculturalisme. Ce dont il ne s'accommode pas, en fait, c'est d'un certain type de multiculturalisme, un multiculturalisme utilisé comme instrument politique visant à occulter le multinationalisme. Mais les deux peuvent très bien aller de pair. Comme l'ont démontré les travaux de Will Kymlicka ${ }^{4}$ la plupart des démocraties libérales forment aujourd'hui des sociétés à la fois polyethniques et multinationales, c'est-à-dire que les sources principales de la diversité culturelle dans ces sociétés sont à chercher soit du côté de l'immigration, soit du côté de l'incorporation de communautés culturelles préexistantes à l'intérieur d'un État plus large, que ce soit par conquête ou par traité.

$+4+$

4. Will KyMLICKA, Multscultural Citizenship, Oxford, Oxford University Press, 1995. Vorr en particulier p. 11-26. 
Le Canada pourrait être un pays officiellement polyethnique et multinational, c'est-à-dire qu'il pourrait reconnaître explicitement les deux sources distinctes de la diversité culturelle sur son territoire, la source polyethnique reposant sur l'immigration et la source multinationale reposant sur les cultures préalablement ou longuement établies sur le territoire, au lieu de subordonner la seconde à la première et de masquer ainsi la différence entre les deux sources de la diversité culturelle. Si une telle politique de multiculturalisme avait vu le jour, il y a longtemps que la cause des nationalistes québécois aurait perdu la plupart de ses éléments justificateurs. Toutefois, Kymlicka croit que la politique canadienne sur le multiculturalisme n'a pas besoin d'une telle différenciation puisque l'intégration linguistique des cultures issues de l'immigration au sein des deux communautés nationales dont se compose le Canada, anglophone et francophone, aurait été en un certain sens présupposée par les législateurs, lesquels auraient par là même implicitement reconnu l'existence du peuple québécois's. Sans vouloir m'engager plus avant dans cette discussion, il me semble qu'il s'agit là d'une lecture pour le moins idéalisante et biaisée de la politique canadienne sur le multiculturalisme: idéalisante parce qu'elle prend pour acquis que l'intégration des immigrants au sein des deux communautés linguistiques officielles du Canada doit forcément être interprétée comme une intégration au sein des deux communautés nationales, ce qui est loin d'être évident compte tenu de la vision de la nation canadienne qui s'exprime dans la politique sur le bilinguisme, comme je l'ai expliqué plus haut; biaisée parce qu'elle néglige l'utilisation politique et partisane du multiculturalisme, dont j’ai également parlé. J'aurais donc tendance à être d'accord avec ceux qui rejettent cette lecture ${ }^{6}$.

\section{Les attentes du Québec à l'endroit des immigrants sont-elles compatibles avec le multiculturalisme?}

Une objection importante pourrait néanmoins être adressée à ceux qui croient que le nationalisme québécois s'accommode plutôt bien du multiculturalisme. Un objecteur éventuel pourrait concéder que le multiculturalisme canadien a du mal à reconnaître la réalité multinationale du Canada. Il pourrait même convenir que la politique canadienne sur le multiculturalisme

$$
+4+
$$

5. Will KYMLICKA, "Le libéralisme er la politisacion de la culture", Michel SEYMOUR [éd.], Une nation peut-elle se donner La constztution de son choxx?, Montrél, Bellarmin, 1995, p. 93-119 (voir p. 96).

6. Je pense en particulier à Michel SEMMOUR, La nation en questron, Montréal, L'Hexagone, 1999, p. 52 et suivantes; et Le pari de la demesure. Lintransigeance canadienne face au Quebec, Montréal, L'Hexagone, 2001, p. 117 et suivantes. 
a été adoptée jusqu'à un certain point à des fins politiques, pour mater le nationalisme québécois. Bref, il pourrait concéder que, jusqu'ici, les nationalistes québécois ont eu parfaitement raison de rejeter le multiculturalisme canadien puisque ce multiculturalisme ne les reconnaissait tout simplement pas en tant que peuple ou banalisait l'existence de celui-ci en le mettant sur le même pied que les cultures issues de l'immigration. Il pourrait cependant contester la thèse selon laquelle le nationalisme québécois s'oppose au multiculturalisme canadien seulement et non au multiculturalisme en tant que tel. L'objecteur pourrait en effet attirer l'attention sur le fait suivant: même si les dirigeants politiques ou les juges de la Cour suprême reconnaissaient explicitement le caractère multinational du Canada, même s'ils reconnaissaient constitutionnellement le Québec comme une nation ou une société distincte, le Québec n'en deviendrait pas pour autant une communauté politique plus ouverte au multiculturalisme. La raison en est que, pour préserver son identité culturelle et linguistique, qui est des plus fragiles dans un contexte nord-américain essentiellement anglophone, le Québec se doit de recourir à des politiques linguistiques qui, si elles ne sont pas entièrement opposées au multiculturalisme, ne peuvent qu'en gêner l'épanouissement. Ces politiques ont inévitablement pour effet de limiter le degré d'ouverture du Québec à la diversité linguistique et culturelle sur son territoire et d'imposer davantage de contraintes à l'intégration des immigrants à leur société d'accueil que dans les autres provinces. Bref, l'objection pourrait être que les politiques linguistiques au Québec constituent un frein à toute véritable politique de multiculturalisme. Une véritable politique de multiculturalisme impliquerait l'abandon de ces politiques linguistiques. Or, un tel abandon ferait du Québec une société de moins en moins "distincte" des autres provinces canadiennes. Si le Québec veut conserver son caractère particulier, il doit préserver ses politiques linguistiques. Ce faisant, il abandonne toute politique de multiculturalisme ou en limite considérablement la portée. En un mot, le nationalisme québécois et le multiculturalisme seraient incompatibles, non pas en raison de la non-reconnaissance par le Canada de sa composition multinationale (et, en particulier, de la nation québécoise) à travers sa politique sur le multiculturalisme, mais en raison du nationalisme québécois lui-même et de ses politiques linguistiques contraignantes.

Que peut-on répondre à une telle objection? En fait, la question est de savoir si les demandes faites par le Québec à l'endroit des immigrants sont légitimes compte tenu de son projet de société; si, en d'autres termes, le Québec est en mesure de mener à bien l'intégration de ses immigrants en conciliant l'ouverture à la diversité culturelle et la préservation de son 
caractère distinct. En tenant compte des normes d'une société pluraliste, libérale et démocratique, qu'est-ce que le Québec est en droit d'attendre exactement des immigrants? De telles attentes sont-elles compatibles avec une politique de multiculturalisme?

Pour clarifier ces questions, il convient de se reporter aux documents officiels. En vue de préciser les relations de l'État québécois avec les nouveaux arrivants, le ministère de l'Immigration et des Communautés culturelles a repris les principaux éléments d'un document publié en 1990 à l'initiative du ministère des Relations avec les citoyens et de l'Immigration, intitulé Énoncé de politique gouvernementale en matière d'immigration et d'intégration, qui engageait l'ensemble des organismes et ministères du gouvernement du Québec à favoriser l'atteinte des objectifs d'intégration. Le document établissait les termes d'un "contrat moral» entre le Québec et les personnes désirant y immigrer, balisant les droits et les devoirs impartis à chacun. Pour l'essentiel, ce document inspire toujours les grandes orientations du ministère de l'Immigration ${ }^{7}$. L'intégration des immigrants est mesurée à l'aune de trois paramètres:

1. Le Québec est une société d'expression française. Le français est la langue officielle et la langue de la vie publique.

2. Le Québec est une société démocratique. Il est un État laïque fondé sur la séparation de l'Église et de l'État et sur une Charte des droits énonçant l'egalité de traitement de tous les citoyens.

3. Le Québec est une société pluraliste qui demeure ouverte à la multiplicité des styles de vie, des valeurs, des opinions et des orientations religieuses, pour autant que ces derniers respectent les lois et les droits reconnus à tous les citoyens.

Essentiellement, l'attente de l'État québécois à l'endroit des immigrants est qu'ils respectent ces trois caractéristiques de la société québécoise. En d'autres termes, pour s'intégrer à son nouveau milieu de vie, le nouvel arrivant doit faire les efforts requis pour apprendre la langue française, qui est la langue de la vie publique, celle "du travail, des communications, du commerce et des affaires", mais aussi "un symbole commun d'appartenance

$$
++4
$$

7. En effet, les princıpes direcceurs de ce "contrat moral s sont repris presque mot pour mot dans plusieurs sections du sire officiel du ministère, dont la section intitulée "Pourquoi choısir le Québec* (http://www.immigration. quebec.gouv.qc.ca/francais/avantages-quebec/societe.html). 
à la société québécoise " ${ }^{8}$; il est également invité à participer pleinement à la vie démocratique québécoise, dans le cadre de ses institutions laïques et en respectant l'égalité de traitement réservée aux citoyens; finalement, il est convié non pas à renoncer à sa langue, à sa culture ou à sa religion, que l'État québécois voit comme un apport et un enrichissement, mais à tenter d'adapter le mieux possible l'héritage culturel dont il est porteur - l'adapter au fait français de la vie publique, mais aussi aux valeurs démocratiques de la société québécoise, notamment en ce qui concerne l'égalité de l'homme et de la femme et l'interdit de toute forme de discrimination ethnique ou raciale. Ces demandes faites par l'État québécois à l'endroit des immigrants sont-elles légitimes? Sont-elles compatibles avec une politique de multiculturalisme?

\section{Le multiculturalisme et la culture publique au Québec}

Commençons notre examen par les demandes relatives à la démocratie et au pluralisme, qui semblent au premier abord les demandes les moins exigeantes ${ }^{9}$. En effet, ces demandes semblent nécessiter davantage d'efforts de la part de l'État québécois (en termes d'investissement dans les mesures d'intégration des immigrants à la vie démocratique et à la vie publique nationale) que des immigrants eux-mêmes. En fait, pour être juste, il faudrait dire que la démocratie et le pluralisme imposent certaines obligations, ou certaines limites à ce qui peut être fait des deux côtés. Du côté des immigrants, une de ces obligations consiste non pas à abandonner leurs convictions morales ou religieuses, mais à accepter les valeurs démocratiques libérales et le pluralisme comme des valeurs propres à la culture politique et à la vie publique du Québec. En d'autres termes, la culture politique publique du Québec, qui est celle d'un État démocratique libéral, doit être distinguée des multiples cultures propres aux groupes religieux et ethniques (provenant ou non de l'immigration). Ce qui est demandé à leurs membres, c'est non pas de renoncer (dans la vie privée ou dans les associations de la vie civile) à leurs allégeances culturelles ou religieuses, mais de les adapter aux normes d'une culture politique commune et publique, qui imposent à tous les membres de la société certains principes de vie (égale liberté de conscience, d'opinion, de religion) sans lesquels un pluralisme raisonnable, c'est-à-dire la coexistence

$$
++4
$$

8. Ministére de l'Éducation dU Québec, "Pourquoi choisir le Québec", htrp://www.immigrationquebec.gouv.qc.ca/francais/avantages-quebec/societe.html.

9. Je m'inspirerai dans cette section et dans la suivante des analyses pénétrantes faites par joseph CARENS de la siruacion des immigrants au Quebec dans le chapitre 5 de son ouvrage Culture, Citzenship, and Community, Oxford, Oxford University Press, 2000. 
pacifique d'une multitude de convictions religieuses et d'allégeances culturelles sur un même territoire, serait à proprement parler impossible ${ }^{10}$.

Mais les valeurs démocratiques libérales et pluralistes qui balisent la latitude accordée aux immigrants quant à la place donnée à leur héritage culturel dans leur société d'accueil imposent réciproquement certaines limites à ce que le Québec est en droit d'attendre d'eux en termes d'intégration, de même qu'elles entraînent certaines obligations de sa part. L'engagement de l'État québécois en faveur du pluralisme lui interdit d'exiger des immigrants qu'ils s'assimilent à la "culture" québécoise, qu'ils partagent, par exemple, la mode vestimentaire, le régime alimentaire, les loisirs, les horaires de travail ou les allégeances religieuses de la majorité. Au contraire, en considérant "le savoir-faire, les compétences, la langue, la culture et la religion des groupes immigrants comme un apport et un enrichissement", l'État québécois s'engage moralement, non seulement à accommoder ces pratiques - c'est-à-dire à adapter ou à modifier le style de vie de la majorité de manière à laisser une place à celui des minorités culturelles là où la chose est moralement acceptable et pratiquement faisable en termes de ressources -, mais également à les promouvoir (au moyen, par exemple, de subventions publiques accordées à certaines communautés pour leurs festivals, leurs associations, leurs magazines, etc.).

Ce type de limitation et d'engagement réciproque du gouvernement du Québec et des communautés culturelles est-il légitime? Est-il compatible avec une politique de multiculturalisme? Je vois difficilement comment on pourrait soutenir le contraire. Tout d'abord, la demande faite aux immigrants de conformer leurs pratiques culturelles aux normes d'une culture publique libérale s'adresse non seulement à eux, mais à tous les membres de la société québécoise, même aux supporteurs d'une religion catholique traditionaliste (qui formait, rappelons-le, une partie importante de la culture québécoise d'avant la Révolution tranquille), dont plusieurs membres entretiennent sans doute encore une certaine hostilité à l'endroit des valeurs démocratiques et pluralistes du Québec moderne ${ }^{11}$. Autrement dit, la demande de conformité à l'endroit d'une culture publique libérale est égali-

$$
+4
$$

10. Sur la distinction encre culcure politique publique ec cultures ad'arrière-plan n, voir John RAWLS, Politzcal Laberalssm, New York, Columbia University Press, 1993, p. 13-14; er The Law of Peoples, Cambridge, Harvard University Press, 1999 , p. $152-156$.

11. Comme on le sait, les commissions scolarres confessionnelles ont été abolies en 1998, et les groupes de pression favorables au maintien des cours d'enseignement catholique ou protestant à l'école doivent recourir à la controversée clause dérogatoire afin de passer outre les principes de la Charte canadienne des droits ec libertés, qui prévoit, comme la Charte québeccoise des droits de la personne, le drott à la liberté et à l'égalité de religion. 
taire et inclusive; elle ne fait de discrimination à l'endroit d'aucun groupe. Ensuite, et surtout, une telle demande n'exige pas que les immigrants se conforment à une culture qui serait spécifiquement québécoise, mais les oblige à respecter des normes, des valeurs et des principes (séparation de l'État et de l'Église, respect des droits humains fondamentaux, égalité démocratique des citoyens) qui gouvernent la vie publique de toutes les démocraties libérales. En d'autres termes, si cette demande est justifiée, ce n'est pas parce qu'il s'agit d'une demande faite par le Québec à titre de "société distincte", mais parce que les valeurs dont elle se réclame sont justes, en ce sens qu'elles forment les conditions les plus favorables à un ordre politique légitime parmi toutes celles connues à ce jour. Un immigrant qui refuserait les valeurs promues par la culture publique du Québec refuserait non pas des valeurs spécifiquement québécoises, mais des valeurs partagées par toutes les démocraties libérales. Ce n'est pas d'un problème particulier d'intégration à la culture québécoise qu'il souffrirait, mais d'un problème d'acceptation des valeurs libérales et démocratiques elles-mêmes ${ }^{12}$.

Par conséquent, un objecteur éventuel pourrait concéder que, parmi les demandes faites par le Québec à l'endroit des immigrants, aucune n'est irrecevable ou ne contrevient à une politique de multiculturalisme. Or, poursuivrait l'objecteur, ces demandes sont telles précisément parce qu'elles exigent peu d'adaptation de la part des immigrants à la "spécificité culturelle" québécoise; autrement dit, elles sont recevables tant que le caractère "distinct» du projet de société québécois n'est pas en cause. Cette situation serait toutefois battue en brèche par les politiques linguistiques du Québec, qui placeraient la société québécoise devant un dilemme: si elle veut conserver son caractère distinct, elle doit absolument préserver ses politiques linguistiques et abandonner toute politique de multiculturalisme puisqu'une véritable politique de multiculturalisme nécessiterait l'abandon de ces politiques linguistiques; si, par contre, le Québec devait choisir la voie du multiculturalisme, le projet de société du Québec n'aurait plus rien de

$$
+4+
$$

12. Jocelyn Maclure parle en ce sens d'adhésion à des " méta-normes " consriturives de la démocratie libérale comme régime politique, lesquelles sont à discinguer des uchoix de soctétén particuliers faits par les citoyens, comme de faire du français la langue de la vie publique. Alors que les choix de société peuvent évoluer au gré des cırconstances ex du jeu des décisions majoricaires, les a méta-normes n sont plus difficilement modifiables en ce qu'elles forment les conditions de possibilité à des choix de sociéré légitimes. Elles peuvent sans doute être interprêtées différemmenc selon les contextes et doivent donc être constamment actualisées, mais elles ne peuvent pas être purement et simplement abolies. Sur ce point, voir Jocelyn MACLURE, * Politique lınguistique ou politique d'intégration? La promotion de la langue dans une communauté polıtique libérale, démocratique et pluraliste», Pierre GeorGeaulT et Michel PaGÉ [éd.], Le franfais, langue de la diverstté québécoise, Montréal, Québec Amérique, 2006, p. 165. 
"distinct», et le Québec devrait renoncer à tous les efforts faits jusqu'à ce jour en vue de préserver son identité linguistique et nationale. Le Québec fait-il vraiment face à un tel dilemme? C'est ce que j'aimerais examiner à présent.

\section{Le multiculturalisme face aux politiques linguistiques québécoises}

Accepter la démocratie et le pluralisme comme principes gouvernant la culture politique et la vie publique au Québec est une chose. Accepter que le français y soit la langue officielle, la langue de la vie publique, en est une autre. Trois objections pourraient être formulées relativement aux politiques linguistiques québécoises ${ }^{13}: 1$ ) Certains aspects de la législation linguistique au Québec, en promouvant l'utilisation du français, restreignent l'utilisation d'autres langues, contrevenant ainsi à la liberté linguistique des citoyens, qui est un droit fondamental; 2) Il est inacceptable de faire de l'acquisition du français comme langue de la vie publique une condition d'intégration à la société québécoise; 3) La politique obligeant les immigrants à éduquer leurs enfants dans des écoles de langue française est inéquitable puisque les anglophones nés au Canada ont le droit d'éduquer leurs enfants dans des écoles de langue anglaise.

Avant de répondre à ces objections, je tiens à rappeler que la cible principale des politiques linguistiques au Québec n'est pas la langue d'origine des immigrants, mais bien la prédominance de l'anglais en Amérique du Nord et l'attrait naturel exercé par cette langue auprès des immigrants, qui rendent la situation du français précaire. Chacun sait que, avant l'adoption de la loi 101, les immigrants non francophones adoptaient massivement l'anglais comme langue d'usage et éduquaient leurs enfants dans cette langue. La loi 101, en obligeant les immigrants à choisir pour leurs enfants une école de langue française, fut une mesure certes contraignante pour eux, mais sans laquelle l'équilibre des forces linguistiques en présence au Québec aurait été sérieusement menacé. L'adoption de la loi 101 était donc de nature défensive; il s'agissait, pour reprendre l'expression de Will Kymlicka, d'une mesure de "protection externe" ${ }^{14}$. Je ne fais ici que mettre en contexte les politiques linguistiques au Québec, sans pour autant les justifier. Pour cela, il faut voir s'il est possible de répondre convenablement aux objections soulevées plus haut.

$$
++
$$

13. Dans ce qui suit, je m'appuierai en partue sur l'analyse proposée par Joseph CARENS, op ctt., p. 124-139.

14. Sur la distunction entre a restriction interne" et "protection externe", voir Will KYMLICKA, Mulncultural Citizenship, p. $35-44$. 
La première de ces objections avait une certaine pertinence au moment où la loi 101 interdisait l'affichage commercial dans toute autre langue que le français. Dans les années 1980, la Cour suprême du Canada avait avisé le gouvernement du Québec que cette disposition de la loi n'était pas conforme à la Charte des droits et libertés. Le gouvernement de l'époque, dirigé par le Parti libéral de Robert Bourassa, avait eu recours à la clause dérogatoire (loi 178) en vue de s'exempter de l'obligation d'adapter la loi 101 aux dispositions de la Charte. Plus tard, sans doute à la suite de la critique faite par les Nations unies au sujet de cette politique linguistique, jugée non conforme aux droits de l'homme, la loi 101 fut amendée et la loi 86, adoptée, ré-autorisant l'utilisation de l'anglais et de toute autre langue dans l'affichage commercial, à la condition que le français soit prédominant. Puisque toutes les communautés linguistiques du Québec peuvent désormais afficher librement dans leur langue, la critique voulant que la promotion du français restreigne l'utilisation des autres langues n'est tout simplement pas fondée. Elle n'est pas fondée, tout d'abord, parce que le fait d'obliger les communautés non francophones à afficher en français n'est plus un frein à l'utilisation de leur propre langue. La loi 86 établit une juste mesure entre l'intérêt légitime des Québécois à préserver l'aspect francophone de l'affichage public et l'intérêt tout aussi légitime des autres communautés linguistiques à afficher dans leur langue, une condition importante pour la vitalité culturelle de ces communautés. La critique voulant que la promotion du français restreigne la liberté linguistique n'est pas fondée pour une deuxième raison, fondamentale. Avant même que n'entre en vigueur la loi 86 , la loi 101 n'interdisait aucunement aux membres des communautés culturelles de parler leur propre langue à la maison ou dans leur communauté. Il ne s'agissait pas d'une mesure législative autoritaire visant à asseoir de manière hégémonique la prédominance du français dans tous les domaines de la vie au Québec, mais uniquement d'une disposition destinée à préserver l'image francophone du Québec dans les signes publics, c'est-à-dire à consolider le français comme langue publique. La loi 101 ne restreignait donc la liberté linguistique que de manière partielle et, pourrait-on dire, mineure: cette limitation se cantonnait strictement aux signes commerciaux. Avant même l'adoption de la loi 86, l'objection à propos de la liberté linguistique était donc discutable. Après l'adoption de la loi 86 , elle perd toute pertinence.

Je passe maintenant à la seconde objection. Est-ce trop demander aux immigrants et aux non-francophones d'adopter le français comme langue de la vie publique (de les obliger, donc, à acquérir cette langue) s'ils veulent s'intégrer à la société québécoise? Comme je l'ai mentionné dans ma réponse 
à la première objection, une certaine idée de neutralité par rapport aux usages linguistiques possède une vertu indéniable; il est mauvais de vouloir contraindre, par des moyens politiques, la liberté linguistique et d'imposer l'usage d'une seule langue. Une pareille position, qui correspond à ce que les anglophones qualifient de "bands off approach to language", impose des limites à ce que les politiques linguistiques sont autorisées à faire. Cette approche possède cependant ses propres limites. Une communauté politique ne peut pas être entièrement neutre par rapport à tout usage linguistique, comme l'a montré Will Kymlicka de manière convaincante ${ }^{15}$. Une communauté politique n'a tout simplement pas d'autre choix que de décider quelle(s) langue(s) sera (seront) utilisée(s) par le gouvernement dans les tribunaux, le parlement, la distribution des services publics et les écoles. À la différence de la séparation de l'État et de l'Église, une totale séparation de l'État et de la culture est une chose impossible ${ }^{16}$. Bref, quand on cherche à tenir compte de ce qui doit moralement être évité (contraindre de manière autoritaire les libertés linguistiques) et de ce qui, pratiquement, ne peut être réalisé (autoriser tous les usages linguistiques), une chose apparaît inévitable: le choix d'une ou de plusieurs langues (à titre de langues officielles, de langues de la vie publique, de langues dans lesquelles seront offerts les services publics, etc.), donc la mise en place de politiques linguistiques qui demeurent à la discrétion des autorités concernées et qui varient généralement en fonction de chaque contexte (c'est-à-dire de l'histoire, des groupes en présence, de la démographie, du degré de concentration linguistique, etc.). En ces domaines, il n'y a pas une seule réponse correcte, pas plus qu'il n'y a de principe ou de procédure qui s'appliquerait à toutes les situations. On sait néanmoins que, si aucune démocratie libérale ne se reconnaît actuellement l'obligation morale d'offrir des services publics dans toutes les langues parlées par les immigrants, toutes se reconnaissent l'obligation morale de leur offrir les ressources nécessaires pour qu'ils puissent maîtriser convenablement la langue de la majorité. L'histoire montre en effet que l'acquisition de la langue de la majorité par les immigrants est une condition indispensable à leur intégration et qu'une "hands off approach to language» serait en ce sens

$$
++4
$$

15. Voir Will KYMLICKA, Multrulrural Citzzenship, p. 110-111.

16. Daniel Weınstock soutient que l'État devrait se comporter à l'égard de la langue d'une manière qui s'écarte le moins possıble de l'idéal de la "neurralicé bienveillante ( " bensgn neglect"). Ses conclusions, que je ne commenterai pas ici, conduisent à une approche formelle et procédurale des politiques linguistiques, à un quasi-divorce de l'Etac et de la langue publique, conclusions qui ne m'ont paru ni convaincantes ni réalistes. Voir Daniel WEINSTOCK, "The Antinomy of Language Policy*, Will KrMLicka ex Alan PaTTEN [éd.], Language Rights and Political Theory, Oxford, Oxford University Press, 2003, p. 250-270. 
désastreuse. En d'autres mots, abandonner les immigrants à leur sort en leur permettant de parler leur langue d'origine comme bon leur semble, mais sans les soutenir activement dans l'apprentissage de la langue de la majorité serait les condamner à l'exclusion et à la marginalité. Les mesures mises en place par le gouvernement québécois pour accueillir les immigrants se conforment parfaitement à ces pratiques courantes dans les démocraties libérales. Qu'estce qu'un immigrant pourrait alors reprocher à l'obligation d'acquérir et de maitriser convenablement le français comme langue publique afin de s'intégrer à la société québécoise?

Un argument pourrait encore être avancé à l'effet que l'État québécois oblige les immigrants non seulement à faire un usage correct du français dans la vie publique, mais à développer un sentiment d'allégeance et d'identification au fait français comme marque distinctive de la société québécoise, et à s'engager en faveur de la survivance et de l'épanouissement de la langue française au Québec. Cependant, rien dans l'application des politiques linguistiques au Québec ne permet de valider une telle supposition. Si l'engagement actif des immigrants en faveur du fait français constitue certainement un souhait partagé par plusieurs ${ }^{17}$, l'État québécois ne peut légitimement en faire une condition de citoyenneté sans violer les deux autres principes (démocratique et pluraliste) sur lesquels s'échafaude la culture publique qu'il promeut. L'exigence pluraliste, si elle est prise au sérieux, doit laisser aux Néo-Québécois, comme aux Québécois d'origine, la liberté de développer eux-mêmes le type de rapport qu'ils désirent entretenir avec le fait français. Pour certains, la langue française n'aura qu'une valeur instrumentale. Pour d'autres, elle aura une valeur expressive, étant indissociable de leur identité. En contexte pluraliste, un État ne peut légitimement imposer le type de sentiment que les citoyens doivent entretenir à l'égard de la langue publique. Les membres de la majorité n'ont d'autre choix que d'espérer que les nouveaux arrivants se solidarisent peu à peu avec un projet de société qui s'est

$$
+\div
$$

17. C'est par exemple le cas de Michel Pagé qui, s’inspirant de la Commission des Érats généraux sur la situation et l'avenir de la langue française au Québec dirigée par Gerald Larose, ou d'intellecruels québécois comme Gérard Bouchard et Jacques Beauchemin, cente de développer pour le Québec un modèle d'intégration des immigrants plus substantiel que le modèle formel ou procédural, lequel n'envisage le rapport à la langue que dans une perspective instrumentale. Selon le modalle qu'il développe, la promocion du fait français en Amérique du Nord devrait constituer une condition de l'intégration des immigrants à la sociécté québécoise. (Voir Michel PAGé, " Propositions pour une approche dynamique de la siruation du français dans l'espace linguistique québécois», Pierre GEORGEAulT et Michel PAGE [éd.], op. cit., p. 36.) Comme le remarque avec justesse Jocelyn Maclure dans le même ouvrage, une celle promotion peur sans doute conscituer un souhait entretenu à l'endtoit des immigrants, mais elle ne doit en aucun cas devenir une responsabilité qui leur incombe s'ils veulent acquérir la citoyenneté québécoise. (Voir Jocelyn MaCLURE, op. cit., p. 162-163.) 
mis en branle avant leur arrivée et qui est intimement associé à la défense de la langue française, sans faire de cette défense une condition de citoyenneté.

S'il y a lieu de croire que l'exigence pluraliste est respectée dans l'application des politiques linguistiques au Québec, le seul argument qui peut encore être invoqué à l'appui de l'opinion voulant que l'acquisition du français soit une condition d'intégration coercitive à la société québécoise est que le gouvernement québécois ne reconnaît pas sur son territoire les deux langues officielles du Canada, l'anglais et le français. En d'autres termes, l'apprentissage du français représenterait une contrainte illégitime pour les immigrants, non pas parce que le Québec ne reconnaitrait pas officiellement leur langue ou qu'il n'offrirait pas de services publics dans leur langue, non pas même parce qu'il les obligerait à développer un sentiment d'allégeance en faveur de la promotion du fait français, mais parce qu'il limiterait les moyens qui leur sont offerts de s'intégrer à la société québécoise en ne leur laissant d'autre option que celle d'apprendre le français, alors que les immigrants d'avant la loi 101 pouvaient opter pour le français ou l'anglais, qui sont les deux langues officielles du Canada. Cette objection revient à soutenir que le traitement des immigrants au Québec est inéquitable en regard de la politique canadienne sur le bilinguisme et en regard, notamment, du traitement particulier dont jouissent les anglophones du Québec par rapport aux nouveaux immigrants en ce qui concerne l'accès à l'école anglaise. Il s'agit, en somme, de la troisième objection, qu'il nous reste à examiner.

Comme je l'ai signalé, l'évaluation des politiques linguistiques adoptées par un Etat doit tenir compte du contexte dans lequel elles s'insèrent. Premièrement, il faut savoir que la politique du bilinguisme au Canada peut être interprétée de différentes manières. La majorité des Canadiens y voient l'expression de ce qu'on appelle le «bilinguisme personnel». Selon cette lecture, tous les citoyens du Canada peuvent utiliser le français ou l'anglais partout où ils se trouvent sur le territoire et sont autorisés (en principe) à recevoir des services publics dans l'une ou l'autre de ces deux langues, ainsi qu'à recevoir une éducation publique dans l'une ou l'autre de ces deux langues si le nombre le justifie. Le gouvernement québécois favorise pour sa part ce qu'on appelle le «bilinguisme territorial ». Selon cette lecture, le Canada est un pays bilingue pour ce qui est des institutions fédérales, mais les institutions sociales étant de juridiction provinciale, chaque province doit être libre de se définir comme une province bilingue ou de choisir l'une des deux langues comme langue officielle. En faisant du français sa langue officielle, c'est la seconde option qu'a choisie le Québec, et il ne viole en cela aucun principe constitutionnel. Rien, en effet, ne le contraint constitutionnelle- 
ment à se définir comme province ayant deux langues officielles, l'anglais et le français.

En second lieu, il est vrai que, en interdisant l'accès aux écoles publiques de langue anglaise aux enfants d'immigrants récents, le Québec les a privés d'un droit qu'ils possédaient avant la loi 101. Certains pourraient voir là une mesure de "restriction interne" au sens où l'entend Will Kymlicka, c'est-à-dire une mesure limitant les droits des membres d'une communauté en vue d'assurer la survie du groupe ${ }^{18}$. Il faut cependant savoir que le droit qu'avaient les immigrants avant la loi 101 était un droit légal, non un droit moral fondamental, qui leur revenait d'emblée. Aucun État n'est moralement obligé d'offrir des services d'éducation dans la langue de leur choix à tous les citoyens à même les fonds publics. Le système mis en place avant la loi 101 ne comportait d'ailleurs aucunement cette possibilité puisqu'il n'offrait le choix qu'entre le français et l'anglais. En annulant la seconde option pour les immigrants, le Québec a tout simplement fait un choix de politique linguistique auquel il était pleinement autorisé, comme l'est toute société démocratique étant donné les ressources dont elle dispose et les besoins qu'elle rencontre. Comme je l'ai écrit plus haut, cette mesure s'explique par la force d'attraction de l'anglais et la situation précaire du français en Amérique du Nord, par l'adoption massive de l'anglais par les immigrants et les menaces qui, avant son adoption, pesaient sur l'équilibre linguistique au Québec. Or, aucune mesure n'a autant contribué que la loi 101 à l'intégration des immigrants à la communauté francophone et, après l'amendement apporté par la loi 86, à la paix linguistique au Québec. Elle a donc été un bienfait pour l'ensemble de la société québécoise. Bien entendu, elle a limité les options offertes aux immigrants, mais en aucun temps elle ne les a privés d'un droit moral fondamental. Comme le souligne Joseph Carens,

[s]i le Québec était un État indépendant sans aucun système d'éducation publique en anglais, personne ne supposerait que les immigrants ont un droit moral à ce que leurs enfants soient éduqués dans une langue autre que celle utilisée par la vaste majorité de la

$$
+++
$$

\footnotetext{
18. Voir Will KYMLLCKA, Mulficultural Citizenship, p. 35-44. Les « restrictions incernes" n'onc un sens pejoratif que dans la version du libéralisme favorisée par Will Kymlicka, fondée sur l'autonomie. D'autres versions du libéralisme, par exemple celles axées sur le pluralisme et la tolérance, comme chez William Galston ou chez John Rawls, sont prêtes à tolérer de telles restrictions si elles sont volontairement acceptées par les membres de la communauté ou si ceux qui ne les acceptent pas peuvent librement quitter leur communauté. Dans le cas des immigrants au Québec, il convient de rappeler que, si l'accès à l'école anglaise est présentement fermé à leurs enfants, rien n'empêche les adultes de s'intégrer, voire de s'assimiler entièrement à la communauté anglophone s'ils le désirent.
} 
population. [...] C'est uniquement parce que le Québec est une partie du Canada [...] et parce que le Québec dispose d'un système d'éducation publique en anglais qu'il apparaît plausible de penser que les immigrants devraient avoir l'option d'une éducation en anglais ${ }^{19}$.

Reste l'objection selon laquelle l'option de l'école anglaise a été fermée aux immigrants, mais non aux anglophones, c'est-à-dire aux enfants de parents dont l'un au moins a reçu une éducation en anglais au Canada, ce qui serait discriminatoire. Si le Québec offre un système d'éducation publique en anglais, il doit l'offrir à tous et non à quelques privilégiés. Que répondre à cela? Tout d'abord, il faut rappeler que l'accès restreint aux écoles anglaises ne vise pas que les immigrants, mais également les francophones du Québec. Si cette politique n'avait ciblé que les immigrants et non les résidants natifs, l'opinion selon laquelle il y aurait là discrimination aurait sans doute été défendable. Mais elle perd toute pertinence à partir du moment où cette mesure traite de la même manière tous ceux dont aucun des parents n'a reçu une éducation en anglais au Canada, qu’ils soient immigrants ou francophones. Cette mesure n'est donc pas négative, elle n'est pas destinée à priver les immigrants d'un droit qui serait offert à tous les autres citoyens; elle est au contraire positive puisqu'elle vise à accommoder une communauté, de langue anglaise, longuement établie sur le territoire. Si le Québec était un État souverain, il pourrait tout bonnement abolir le système d'éducation publique en anglais, traitant ainsi tous les citoyens de la même manière. En faisant cela, il porterait toutefois préjudice aux membres de la communauté anglophone. La justice n'exige pas que tous soient traités exactement de la même manière, mais que des situations semblables soient traitées de manière semblable et des situations différentes, de manière différente. Or, les membres des minorités longuement établies sur un territoire ont des besoins et des intérêts propres, différents de ceux des immigrants récents, qui doivent être pris en compte. Si, comme je l'ai déjà mentionné, les démocraties libérales ne se reconnaissent aucune obligation morale à desservir tous les immigrants dans leur langue (bien qu'il convienne de considérer le cas de chaque langue à la lumière des ressources disponibles, des circonstances et des besoins exprimés), l'obligation morale devient beaucoup plus forte à l'endroit des minorités de longue date, souvent concentrées territorialement, qui ont un

$$
+4
$$

19. Joseph CARENS, op ctt., p. 30. 
intérêt beaucoup plus marqué à se voir offrir des services publics et un système d'éducation publique dans leur langue. C'est précisément en vue de rencontrer cette obligation morale que le Québec maintient un système d'éducation publique en anglais, lequel vise essentiellement à satisfaire les besoins de la minorité anglophone du Québec.

Que conclure de ces réponses aux trois objections pouvant être formulées par rapport aux politiques linguistiques du Québec? À mon sens, il faut conclure, premièrement, que ces objections ne sont pas fondées et que les politiques linguistiques du Québec sont raisonnables et légitimes lorsqu'on les mesure à l'aune des standards de justice d'une société libérale et démocratique. On doit conclure, en second lieu, qu'elles sont parfaitement compatibles avec une politique de multiculturalisme. En d'autres termes, le Québec ne fait aucunement face au dilemme présenté précédemment, qui l'obligerait à abandonner ses politiques linguistiques s'il souhaite maintenir une politique de multiculturalisme, ou à abandonner une telle politique s'il veut conserver son identité nationale. Le type d'intégration et d'adaptation exigé des immigrants par le Québec se compare parfaitement à celui exigé par la plupart des démocraties libérales, y compris le Canada. Les contraintes imposées aux immigrants par les politiques linguistiques québécoises ne violent aucun droit moral fondamental; elles sont ni plus ni moins coercitives que celles imposées par la plupart des démocraties libérales. Elles découlent d'un choix (linguistique) que nos intuitions concernant la justice et l'égalité de traitement autorisent moralement, et que doit faire une société en fonction des ressources mises à sa disposition, de son histoire, de sa composition démographique et de ses besoins.

\section{CONCLUSION}

J'ai tenté dans cette étude de montrer que le nationalisme québécois ne s'oppose en rien à une politique de multiculturalisme. Dans la première partie, j'ai fait valoir que ce que le nationalisme québécois rejette est non le multiculturalisme en tant que tel, mais le type de multiculturalisme promu à l'intérieur de la fédération canadienne, qui peine à reconnaître la composition multinationale du Canada et, en conséquence, l'existence d'une nation québécoise. Dans la seconde partie, j'ai voulu répondre à l'objection selon laquelle nationalisme québécois et multiculturalisme seraient des options conflictuelles, non en raison de la politique canadienne sur le multiculturalisme, mais à cause du nationalisme québécois lui-même, qui, en voulant préserver l'identité nationale des Québécois, gênerait l'épanouissement d'une véritable politique de multiculturalisme et limiterait le degré d'ouverture du 
Québec à la diversité culturelle. J'ai montré que cette position n'est pas fondée pour deux raisons. Tout d'abord, parce que la culture publique du Québec est une culture libérale, démocratique et pluraliste, et qu'à ce titre elle est entièrement ouverte à la diversité culturelle. Ensuite, parce que les politiques linguistiques du Québec sont parfaitement compatibles avec le multiculturalisme et que la plupart des objections qui sont formulées à leur endroit ne résistent pas à un examen critique attentif. En somme, l'identité nationale que cherche à promouvoir le nationalisme québécois au moyen de ses politiques linguistiques ou de ses politiques en matière d'immigration n'est pas différente de celle promue par la plupart des démocraties libérales à travers le monde. Il ne s'agit pas d'une identité "dense", fondée sur certaines valeurs (morales ou religieuses, par exemple) qui seraient constitutives de l'identité des membres d'une nation ethnique ou culturelle. L'identité en jeu touche plutôt la communauté politique dans son ensemble et consiste en un attachement à la langue, aux institutions publiques et à l'histoire commune. Telle est la nature de l'«identité nationale " que met de l'avant le nationalisme québécois, qui n'est pas très différente de l'identité nationale américaine, française ou britannique: une identité fondée sur la langue, sur les institutions publiques et sur l'histoire commune. Une telle identité est "mince» en ce sens qu'elle est parfaitement compatible avec le pluralisme et qu'elle demeure ouverte à la diversité des cultures, des conceptions du monde et des opinions morales ou religieuses (au sujet de l'avortement, de la peine de mort, de l'euthanasie, etc.). Si le type d'identité nationale que cherche à promouvoir le nationalisme québécois est bien celui que je viens de décrire, il faut conclure que celui-ci n'est ni plus ni moins compatible avec le multiculturalisme que ne le sont la plupart des démocraties libérales à travers le monde. 